July 3, 2021

ETH-TH/95-30

NSF-ITP-95-155

\title{
2-Loop Supersymmetric Renormalization Group Equations Including R-parity Violation and Aspects of Unification
}

\author{
Herbi Dreiner ${ }^{1,2 *}$ and Heath Pois ${ }^{3,4}$ \\ ${ }^{1}$ Theoretische Physik, ETH-Hönggerberg, CH-8093 Zürich, Switzerland \\ ${ }^{2}$ ITP, UC Santa Barbara, Santa Barbara, CA 93106-4030, USA \\ 3 Therma-Wave, 47320 Mission Falls Ct, Freemont, CA 94539, USA \\ ${ }^{4}$ Davis Institute for High Energy Physics, Dept. of Physics, U.C. Davis, CA 95616, USA
}

\begin{abstract}
We present the complete 2-loop renormalization group equations of the supersymmetric standard model. We thus explicitly include the full set of $R$-parity violating couplings, including $\kappa_{i} L_{i} H_{2}$. We use these equations to do a first study of (a) gauge coupling unification, (b) bottom-tau unification, (c) the fixpoint structure of the top quark Yukawa coupling, and (d) two-loop bounds from perturbative unification. We find significant shifts which can be larger than the effect from the top quark Yukawa coupling. The value of $\alpha_{3}\left(M_{Z}\right)$ can change by $\pm 5 \%$. The $\tan \beta$ region for bottom-tau unification and for the top quark IR quasi fixed point structure is significantly increased. For heavy scalar fermion masses $\mathcal{O}(1 \mathrm{TeV})$ the limits on the $\Delta L \neq 0$ operators from perturbative unification are competitive with the indirect laboratory bounds. The two-loop correction to the bound on the $\Delta B \neq 0$ opertor is $+15 \%$.
\end{abstract}

\section{Introduction}

The most compelling indication for supersymmetry is the unification of the gauge coupling constants. This has been thoroughly investigated in the literature [1]-[4], mainly in the context of the minimal supersymmetric standard model (MSSM). In all cases it was assumed that R-parity

$$
R_{p}=(-)^{3 B+L+2 S}
$$

*Permanent address as of February $5^{t h}, 1996$, Rutherford Appleton Laboratory, Chilton, Didcot, Oxon, OX11 0QX, UK. 
is conserved. However, $R_{p}$ is imposed ad hoc: there is no experimental or theoretical justification. $R_{p}$ is sufficient but not necessary to guarantee the stability of the proton. Other discrete [5] or gauge symmetries are equally possible [6, 7]. Strict cosmological bounds based on GUT-scale baryogenesis have been proposed [8] but these have been shown to be strongly model dependent [9]. The best motivation for R-parity is that it offers a good candidate for as yet undetected cold dark matter. However, since this has not directly been observed R-parity violation $\left(R_{p}\right)$ should be considered on equal footing with conserved R-parity in supersymmetric model building and especially in collider searches [10, 11].

Throughout this paper we allow for $R_{p}$. Thus we consider the additional terms in the superpotential

$$
W_{\not R p}=\lambda_{i j k} L_{i} L_{j} \bar{E}_{k}+\lambda_{i j k}^{\prime} L_{i} Q_{j} \bar{D}_{k}+\lambda_{i j k}^{\prime \prime} \bar{U}_{i} \bar{D}_{j} \bar{D}_{k}+\kappa_{i} L_{i} H_{2}
$$

We have used superfield notation and the fields have the $G_{S M}=S U(3)_{c} \times S U(2)_{L} \times U(1)_{Y}$ quantum numbers

$$
\begin{aligned}
& L: \quad\left(1,2,-\frac{1}{2}\right), \quad \bar{E}:(1,1,1), \quad Q:\left(3,2, \frac{1}{6}\right), \quad \bar{U}:\left(3,1, \frac{2}{3}\right), \\
& \bar{D}:\left(3,1,-\frac{1}{3}\right), \quad H_{1}:\left(1,2,-\frac{1}{2}\right), \quad H_{2}:\left(1,2, \frac{1}{2}\right) .
\end{aligned}
$$

$i, j, k$ are generation indices. $\lambda_{i j k}$ is anti-symmetric in the first two indices and $\lambda_{i j k}^{\prime \prime}$ is antisymmetric in the last two.

Models for unification are typically constructed at very high energies, such as grand unified theories (GUTs). Such models can predict the absolute or relative size of parameters at the unification scale. In order to compare the predictions from such models with the low-energy data we must employ the renormalization group ( $R G)$. Recently there have been several studies of the RG properties of the $R_{p}$-Yukawa couplings at one-loop [12]- [17]. These have been used to place first (weak) bounds on several of the higher generation operators via unitarity constraints [13]- [15]. They have also been used to compare $R_{p}$-GUT predictions with the low-energy data [16, 17].

The main interest in the evolution of the gauge coupling constants is whether they unify at a high scale, e.g. grand-unified or string. The $R_{p}$-Yukawa couplings have to date been neglected in this context, mainly because the effect was expected to be small. However, as we show the effect can be larger than the contribution from the top quark Yukawa, $\lambda_{t}$, which can not be neglected. The main reason is that the higher generation couplings are only weakly bound if at all 14, 13, 18. Also, the bounds as presented are usually scaled with the mass dependent factor $(\tilde{m} / 100 \mathrm{GeV})$ and for SUSY masses of order $1 \mathrm{TeV}$ the bound is typically weaker than the bound from perturbative unification. The strictest bounds for a mass of $0.1(1) \mathrm{TeV}$ for the couplings that we will be considering are

$$
\lambda_{323}<0.09(0.9), \quad \lambda_{333}^{\prime}<0.45(4.5)^{*}, \quad \lambda_{323}^{\prime \prime}<1.25 .
$$

\footnotetext{
${ }^{1}$ B: Baryon number, L: Lepton number, S: Spin.
} 
At $1 \mathrm{TeV}$ the bound on $\lambda_{323}$ [11] is almost identical to the perturbative limit obtained below in Section 3. The bound on $\lambda_{333}^{\prime}$ [18] at $1 \mathrm{TeV}$, indicated by an asterisk is obtained by scaling and as such is meaningless since perturbation theory breaks down for such large values. The appropriate bound is thus the perturbative limit. The bound on $\lambda_{323}^{\prime \prime}$ is the bound from perturbative unification [14, 13]. We shall thus explore all three couplings to the pertrubative limit.

We propose to investigate the effect of $\not R_{p}$ on the unification of the gauge coupling constants. The Yukawa couplings only enter at the 2-loop level. We thus extend previous work and present the full two-loop renormalization group equations for $\not R_{p}$ in Section 2 . We then focus on the applications of these equations in Section 3. We investigate the unification of the gauge couplings, bottom-tau unification, and the fixed-point structure of $\lambda_{t}$. We shall perform three case studies, one dominant operator from $L L \bar{E}, L Q \bar{D}$, and $\bar{U} \bar{D} \bar{D}$ respectively, to illustrate the possible $\not R_{p}$ effects. Given the laboratory bounds (1.4), the third generation couplings can be the largest and we shall focus on them for maximum effect. However, it is not necessarily the theoretical expectation that the third generation couplings dominate. If the Standard Model Yukawas and the $\ell_{p}$-Yukawas have a common physical origin, a symmetry, then we expect this symmetry to distinguish between a Higgs superfield and the lepton doublet superfields. If the hierarchy in the Yukawa couplings is determined by the structure of the broken symmetry we would expect the resulting hierarchy to have a different flavour structure for the Higgs couplings then for the purely matter-field couplings [6, 7].

When studying the RG evolution of the gauge couplings the central interest is unification. Does it make sense to discuss $R_{p}$ in the context of unification? In order to avoid rapid proton decay we must require a symmetry which treats quarks and leptons differently. This is counter to any expectation from GUTs where they are in common multiplets. All the same, several supersymmetric grand unified models have been constructed with a low-energy R-parity violating superpotential [19, 20, 16, 17]. These models transfer the extreme mass splitting in the Higgs sector to an asymmetry in the quark-lepton multiplet via $\mathrm{LH}_{2}$ mixing. In order to keep this mixing small it is generated through a broken symmetry. They have no further fine-tuning and the proton decay rate is consistent with experiment. It is thus highly relevant to consider the effects of $\not R_{p}$ on gauge coupling unification.

In string theories there is no preference for grand unification, and unification may very well be obtained with a non-simple group such as $G_{S M}$. As discussed in detail in Ref. 21], there is then also no preference for $R_{p}$ over for example baryon parity $B_{p}=(-)^{3 B+2 S}$. The authors obtain several models with either $R_{p}$ or $\ell_{p}$. The main question in string unification is whether the correct value of $\sin ^{2} \theta_{W}$ can be obtained. Again, in this context the RGEs for the $\not R_{p}$-Yukawa couplings must be considered.

In GUTs the prediction $m_{b}\left(M_{U}\right)=m_{\tau}\left(M_{U}\right)$ has been very successful [22, 23]. In the MSSM if one requires the Yukawa couplings to unify this greatly reduces the allowed region of the (supersymmetric) parameters. In particular one obtains a strict relation between the 
running top mass $m_{t}\left(m_{t}\right)$ and the ratio of the vacuum expectation values $\tan \beta$ [1] [ [] Given the observed top quark mass [24] this results in a prediction for $\tan \beta$. How general is this prediction? The third generation $\not R_{p}$-couplings enter the evolution of $m_{t}, m_{b}$, and $m_{\tau}$ at one loop and can thus have a large effect. Thus if we allow for $\not_{p}$ we expect the strict predictions of the MSSM to be modified. In Section 3.2 we shall analyze this affect and determine a new $\tan \beta$ solution for bottom-tau unification.

With the recent discovery of the top quark we have determined all the fermion masses in the Standard Model. It seems that the top quark mass is special. There has recently been much work to predict the fermion masses at the weak scale from a simple symmetry structure at the unification scale 25, 23]. It is possible that the fermion mass structure is determined by a broken symmetry [26] where only the top-quark Yukawa coupling is allowed by the symmetry at tree-level. It's value is put in by hand, presumably of order one. The other couplings are then determined dynamically through the symmetry breaking model. Given such a model, we would then still require a prediction for the top-quark Yukawa coupling. An intriguing possibility is that this Yukawa coupling is given by an infra-red (quasi) fix point [27]. The low-energy value then depends only very weakly on the high-energy initial value; the exact opposite of a fine-tuning problem. In supersymmetric GUTs with bottom-tau unification one typically requires large values of $\lambda_{t} \sim 1$ close to the IR quasi fixed-point. This has been studied in detail in Refs. 23, 1, 3, 28, 36. In the MSSM this is only attained for a small range of parameters, in particular $\tan \beta$. We investigate the effect of the $\not R_{p}$-couplings on this scenario in Section 3.4. Similar to the case of bottom-tau unification, in Section 3.3 we find a new $\tan \beta$ solution with fixed-point structure.

\section{Renormalization Group Equations}

We apply the work of Martin and Vaughn (MV) [29] to the superpotential (2.2). We shall closely follow their notation. At this point we retain the coupling $\kappa_{i} L_{i} H_{2}$ to be most general and defer a discussion to Section 2.4. We denote the $G_{S M}$ gauge couplings by

$$
g_{3}, \quad g_{2}, \quad g_{1}
$$

The chiral superfields are given in (1.3). In Appendix A we have collected several useful group theoretical formulas pertaining to $G_{S M}$ and the above field content. Here we mention that for $U(1)_{Y}$ we use the normalization as in grand unified theories and thus use $g_{1} \rightarrow g_{Y}$. See the appendix for more details. We define our notation for the Yukawa couplings via the superpotential including all $\not R_{p}$ terms.

$$
\begin{aligned}
W= & \left(\mathbf{Y}_{E}\right)_{i j} L_{i} H_{1} \bar{E}_{j}+\left(\mathbf{Y}_{D}\right)_{i j} Q_{i} H_{1} \bar{D}_{j}+\left(\mathbf{Y}_{U}\right)_{i j} Q_{i} H_{2} \bar{U}_{j} \\
& +\left(\boldsymbol{\Lambda}_{E^{k}}\right)_{i j} L_{i} L_{j} \bar{E}_{k}+\left(\boldsymbol{\Lambda}_{D^{k}}\right)_{i j} L_{i} Q_{j} \bar{D}_{k}+\left(\boldsymbol{\Lambda}_{U^{i}}\right)_{j k} \bar{U}_{i} \bar{D}_{j} \bar{D}_{k} \\
& +\mu H_{1} H_{2}+\kappa^{i} L_{i} H_{2} .
\end{aligned}
$$


We have introduced the twelve $3 \times 3$ matrices

$$
\mathbf{Y}_{E}, \quad \mathbf{Y}_{D}, \quad \mathbf{Y}_{U}, \quad \Lambda_{E^{k}}, \quad \Lambda_{D^{k}}, \quad \Lambda_{U^{i}}
$$

for all the Yukawa couplings. This implies the following conventions

$$
\begin{aligned}
Y_{L_{i} L_{j} \bar{E}_{k}} & =Y_{L_{i} \bar{E}_{k} L_{j}}=Y_{\bar{E}_{k} L_{i} L_{j}}=\left(\boldsymbol{\Lambda}_{E^{k}}\right)_{i j}, \\
Y_{L_{j} L_{i} \bar{E}_{k}} & =Y_{L_{j} \bar{E}_{k} L_{i}}=Y_{\bar{E}_{k} L_{j} L_{i}}=\left(\boldsymbol{\Lambda}_{E^{k}}\right)_{j i}=-\left(\boldsymbol{\Lambda}_{E^{k}}\right)_{i j}, \\
Y_{\bar{U}_{i} \bar{D}_{j} \bar{D}_{k}} & =Y_{\bar{D}_{j} \bar{U}_{i} \bar{D}_{k}}=Y_{\bar{D}_{j} \bar{D}_{k} \bar{U}_{i}}=\left(\boldsymbol{\Lambda}_{U^{i}}\right)_{j k}, \\
Y_{\bar{U}_{i} \bar{D}_{k} \bar{D}_{j}} & =Y_{\bar{D}_{k} \bar{U}_{i} \bar{D}_{j}}=Y_{\bar{D}_{k} \bar{D}_{j} \bar{U}_{i}}=\left(\boldsymbol{\Lambda}_{U^{i}}\right)_{k j}=-\left(\boldsymbol{\Lambda}_{U^{i}}\right)_{j k} .
\end{aligned}
$$

We now in turn study the dimensionless couplings and then briefly also discuss the mass terms $\mu, \kappa_{i}$. We do not here consider the soft-breaking terms.

\subsection{Gauge Couplings}

The renormalization group equations for the gauge couplings are given by

$$
\frac{d}{d t} g_{a}=\frac{g_{a}^{3}}{16 \pi^{2}} B_{a}^{(1)}+\frac{g_{a}^{3}}{\left(16 \pi^{2}\right)^{2}}\left[\sum_{b=1}^{3} B_{a b}^{(2)} g_{b}^{2}-\sum_{x=u, d, e}\left(C_{a}^{x} \operatorname{Tr}\left(\mathbf{Y}_{x}^{\dagger} \mathbf{Y}_{x}\right)+A_{a}^{x} \sum_{i=1}^{3} \operatorname{Tr}\left(\boldsymbol{\Lambda}_{x_{i}}^{\dagger} \boldsymbol{\Lambda}_{x_{i}}\right)\right)\right]
$$

The coefficients $B_{a}, B_{a b}$, and $C_{a}^{x}$ have been given previously [30 and for completeness we present them in the appendix. The $\not R_{p}$-effects on the running of the gauge couplings are new, we obtain

$$
A_{a}^{u, d, e}=\left(\begin{array}{ccc}
12 / 5 & 14 / 5 & 9 / 5 \\
0 & 6 & 1 \\
4 & 4 & 0
\end{array}\right) .
$$

This completes the equations for the running of the gauge coupling constants at two-loop.

\subsection{Yukawa Couplings}

In general the renormalization group equations for the Yukawa couplings are given by [29]

$$
\frac{d}{d t} Y^{i j k}=Y^{i j p}\left[\frac{1}{16 \pi^{2}} \gamma_{p}^{(1) k}+\frac{1}{\left(16 \pi^{2}\right)^{2}} \gamma_{p}^{(2) k}\right]+(k \leftrightarrow i)+(k \leftrightarrow j),
$$

and the one- and two-loop anomalous dimensions are

$$
\begin{aligned}
\gamma_{i}^{(1) j}= & \frac{1}{2} Y_{i p q} Y^{j p q}-2 \delta_{i}^{j} \sum_{a} g_{a}^{2} C_{a}(i) \\
\gamma_{i}^{(2) j}= & -\frac{1}{2} Y_{i m n} Y^{n p q} Y_{p q r} Y^{m r j}+Y_{i p q} Y^{j p q} \sum_{a} g_{a}^{2}\left[2 C_{a}(p)-C_{a}(i)\right] \\
& +2 \delta_{i}^{j} \sum_{a} g_{a}^{2}\left[g_{a}^{2} C_{a}(i) S_{a}(R)+2 \sum_{b} C_{a}(i) C_{b}(i)-3 g_{a}^{2} C_{a}(i) C\left(G_{a}\right)\right]
\end{aligned}
$$


See the appendix for the definition of the group theoretical quantities $C_{a}(f), S_{a}(R)$, and $C\left(G_{a}\right)$. We now first give the explicit version of Eq.(2.10) for the matrices (2.3) in terms of the anomalous dimensions, and then we present the explicit forms for $\gamma_{f_{i}}^{(1) f_{j}}$, and $\gamma_{f_{i}}^{(2) f_{j}}$.

\subsubsection{RG-Equations}

The RGEs Yukawa couplings are given by

$$
\begin{aligned}
\frac{d}{d t}\left(\mathbf{Y}_{E}^{*}\right)_{i k} & =\left(\mathbf{Y}_{E}^{*}\right)_{i p} \Gamma_{E_{p}}^{E_{k}}+\left(\mathbf{Y}_{E}^{*}\right)_{i k} \Gamma_{H_{1}}^{H_{1}}+\left(\boldsymbol{\Lambda}_{E^{k}}^{*}\right)_{i p} \Gamma_{L_{p}}^{H_{1}}+\left(\mathbf{Y}_{E}^{*}\right)_{p k} \Gamma_{L_{p}}^{L_{i}} \\
\frac{d}{d t}\left(\mathbf{Y}_{D}^{*}\right)_{i k} & =\left(\mathbf{Y}_{D}^{*}\right)_{i p} \Gamma_{D_{p}}^{D_{k}}+\left(\mathbf{Y}_{D}^{*}\right)_{i k} \Gamma_{H_{1}}^{H_{1}}+\left(\boldsymbol{\Lambda}_{D^{k}}^{*}\right)_{p i} \Gamma_{L_{p}}^{H_{1}}+\left(\mathbf{Y}_{D}^{*}\right)_{p k} \Gamma_{Q_{p}}^{Q_{i}} \\
\frac{d}{d t}\left(\mathbf{Y}_{U}^{*}\right)_{i k} & =\left(\mathbf{Y}_{U}^{*}\right)_{i p} \Gamma_{U_{p}}^{U_{k}}+\left(\mathbf{Y}_{U}^{*}\right)_{i k} \Gamma_{H_{2}}^{H_{2}}+\left(\mathbf{Y}_{U}^{*}\right)_{p k} \Gamma_{Q_{p}}^{Q_{k}} \\
\frac{d}{d t}\left(\boldsymbol{\Lambda}_{E^{k}}^{*}\right)_{i j} & =\left(\boldsymbol{\Lambda}_{E^{p}}^{*}\right)_{i j} \Gamma_{E_{p}}^{E_{k}}+\left(\boldsymbol{\Lambda}_{E^{k}}^{*}\right)_{i p} \Gamma_{L_{p}}^{L_{j}}+\left(\mathbf{Y}_{E}^{*}\right)_{i k} \Gamma_{H_{1}}^{L_{j}}+\left(\boldsymbol{\Lambda}_{E^{k}}^{*}\right)_{p j} \Gamma_{L_{p}}^{L_{i}}+\left(\mathbf{Y}_{E}^{*}\right)_{j k} \Gamma_{H_{1}}^{L_{i}}, \\
\frac{d}{d t}\left(\boldsymbol{\Lambda}_{D^{k}}^{*}\right)_{i j} & =\left(\boldsymbol{\Lambda}_{D^{p}}^{*}\right)_{i j} \Gamma_{D_{p}}^{D_{k}}+\left(\boldsymbol{\Lambda}_{D^{k}}^{*}\right)_{i p} \Gamma_{Q_{p}}^{Q_{j}}+\left(\boldsymbol{\Lambda}_{D^{k}}^{*}\right)_{p j} \Gamma_{L_{p}}^{L_{j}}+\left(\mathbf{Y}_{D}^{*}\right)_{j k} \Gamma_{H_{1}}^{L_{i}} \\
\frac{d}{d t}\left(\boldsymbol{\Lambda}_{U^{i}}^{*}\right)_{j k} & =\left(\boldsymbol{\Lambda}_{U^{i}}^{*}\right)_{j p} \Gamma_{D_{p}}^{D_{k}}+\left(\boldsymbol{\Lambda}_{U^{i}}^{*}\right)_{p k} \Gamma_{D_{p}}^{D_{j}}+\left(\boldsymbol{\Lambda}_{U^{p}}^{*}\right)_{j k} \Gamma_{U_{p}}^{U_{i}}
\end{aligned}
$$

At two-loop the anomalous dimensions are given by

$$
\Gamma_{f_{i}}^{f_{j}}=\frac{1}{16 \pi^{2}} \gamma_{f_{i}}^{(1) f_{j}}+\frac{1}{\left(16 \pi^{2}\right)^{2}} \gamma_{f_{i}}^{(2) f_{j}}
$$

As we discuss below in section 2.4 in many cases of interest the anomalous dimension $\Gamma_{L_{i}}^{H_{1}}$ vanishes.

\subsubsection{Anomalous Dimensions}

The one-loop anomalous dimensions are given by

$$
\begin{aligned}
\gamma_{L_{i}}^{(1) L_{j}} & =\left(\mathbf{Y}_{E} \mathbf{Y}_{E}^{\dagger}\right)_{i j}+\left(\boldsymbol{\Lambda}_{E^{q}} \boldsymbol{\Lambda}_{E^{q}}^{\dagger}\right)_{i j}+3\left(\boldsymbol{\Lambda}_{D^{q}} \boldsymbol{\Lambda}_{D^{q}}^{\dagger}\right)_{i j}-\delta_{i}^{j}\left(\frac{3}{10} g_{Y}^{2}+\frac{3}{2} g_{2}^{2}\right), \\
\gamma_{E_{i}}^{(1) E_{j}} & =2\left(\mathbf{Y}_{E}^{\dagger} \mathbf{Y}_{E}\right)_{j i}+\operatorname{Tr}\left(\boldsymbol{\Lambda}_{E^{i}} \boldsymbol{\Lambda}_{E^{j}}^{\dagger}\right)-\delta_{i}^{j}\left(\frac{6}{5} g_{Y}^{2}\right), \\
\gamma_{Q_{i}}^{(1) Q_{j}} & =\left(\mathbf{Y}_{D} \mathbf{Y}_{D}^{\dagger}\right)_{i j}+\left(\mathbf{Y}_{U} \mathbf{Y}_{U}^{\dagger}\right)_{i j}+\left(\boldsymbol{\Lambda}_{D^{q}}^{\dagger} \boldsymbol{\Lambda}_{D^{q}}\right)_{j i}-\delta_{i}^{j}\left(\frac{1}{30} g_{Y}^{2}+\frac{3}{2} g_{2}^{2}+\frac{8}{3} g_{3}^{2}\right), \\
\gamma_{D_{i}}^{(1) D_{j}} & \left.=2\left(\mathbf{Y}_{D}^{\dagger} \mathbf{Y}_{D}\right)_{j i}+2 \operatorname{Tr}\left(\boldsymbol{\Lambda}_{D^{i}} \boldsymbol{\Lambda}_{D^{j}}^{\dagger}\right)+2\left(\boldsymbol{\Lambda}_{U^{q}} \boldsymbol{\Lambda}_{U^{q}}^{\dagger}\right)_{i j}-\delta_{i}^{j}\left(\frac{2}{15} g_{Y}^{2}+\frac{8}{3} g_{3}^{2}\right)\right), \\
\gamma_{U_{i}}^{(1) U_{j}} & \left.=2\left(\mathbf{Y}_{U}^{\dagger} \mathbf{Y}_{U}\right)_{i j}+\operatorname{Tr}\left(\boldsymbol{\Lambda}_{U^{i}} \boldsymbol{\Lambda}_{U^{j}}^{\dagger}\right)-\delta_{i}^{j}\left(\frac{8}{15} g_{Y}^{2}+\frac{8}{3} g_{3}^{2}\right)\right), \\
\gamma_{H_{1}}^{(1) H_{1}} & =\operatorname{Tr}\left(3 \mathbf{Y}_{D} \mathbf{Y}_{D}^{\dagger}+\mathbf{Y}_{E} \mathbf{Y}_{E}^{\dagger}\right)-\left(\frac{3}{10} g_{Y}^{2}+\frac{3}{2} g_{2}^{2}\right), \\
\gamma_{H_{2}}^{(1) H_{2}} & =3 \operatorname{Tr}\left(\mathbf{Y}_{U} \mathbf{Y}_{U}^{\dagger}\right)-\left(\frac{3}{10} g_{Y}^{2}+\frac{3}{2} g_{2}^{2}\right), \\
\gamma_{L_{i}}^{(1) H_{1}} & =3\left(\boldsymbol{\Lambda}_{D^{q}} \mathbf{Y}_{D}^{*}\right)_{i q}+\left(\boldsymbol{\Lambda}_{E^{q}} \mathbf{Y}_{E}^{*}\right)_{i q} .
\end{aligned}
$$


For the two-loop anomalous dimensions we write

$$
\gamma_{f_{i}}^{(2) f_{j}}=\left(\gamma_{f_{i}}^{(2) f_{j}}\right)_{\text {yukawa }}+\left(\gamma_{f_{i}}^{(2) f_{j}}\right)_{g-y}+\left(\gamma_{f_{i}}^{(2) f_{j}}\right)_{\text {gauge }} .
$$

These correspond respectively to the three terms of (2.12). These are given explicitly below. The pure gauge two-loop anomalous dimensions are given by

$$
\begin{aligned}
& \left(\gamma_{L_{i}}^{(2) L_{j}}\right)_{\text {gauge }}=\delta_{i}^{j}\left(\frac{15}{4} g_{2}^{4}+\frac{207}{100} g_{Y}^{4}+\frac{9}{10} g_{2}^{2} g_{Y}^{2}\right), \\
& \left(\gamma_{E_{i}}^{(2) E_{j}}\right)_{\text {gauge }}=\delta_{i}^{j} \frac{234}{25} g_{Y}^{4}, \\
& \left(\gamma_{Q_{i}}^{(2) Q_{j}}\right)_{\text {gauge }}=\delta_{i}^{j}\left(-\frac{8}{9} g_{3}^{4}+\frac{15}{4} g_{2}^{4}+\frac{199}{900} g_{Y}^{4}+8 g_{3}^{2} g_{2}^{2}+\frac{8}{45} g_{3}^{2} g_{Y}^{2}+\frac{1}{10} g_{2}^{2} g_{Y}^{2}\right), \\
& \left(\gamma_{D_{i}}^{(2) D_{j}}\right)_{\text {gauge }}=\delta_{i}^{j}\left(-\frac{8}{9} g_{3}^{4}+\frac{202}{225} g_{Y}^{4}+\frac{32}{45} g_{3}^{2} g_{Y}^{2}\right), \\
& \left(\gamma_{U_{i}}^{(2) U_{j}}\right)_{\text {gauge }}=\delta_{i}^{j}\left(-\frac{8}{9} g_{3}^{4}+\frac{856}{225} g_{Y}^{4}+\frac{128}{45} g_{3}^{2} g_{Y}^{2}\right), \\
& \left(\gamma_{H_{1}}^{(2) H_{1}}\right)_{\text {gauge }}=\left(\gamma_{H_{2}}^{(2) H_{2}}\right)_{\text {gauge }}=\left(\gamma_{L_{i}}^{(2) L_{j}}\right)_{\text {gauge }}, \\
& \left(\gamma_{L_{i}}^{(2) H_{1}}\right)_{\text {gauge }}=0,
\end{aligned}
$$

The mixed gauge-Yukawa two-loop anomalous dimensions are given by

$$
\begin{aligned}
\left(\gamma_{L_{i}}^{(2) L_{j}}\right)_{g-y}= & \left(16 g_{3}^{2}-\frac{2}{5} g_{Y}^{2}\right)\left(\boldsymbol{\Lambda}_{D^{q}} \boldsymbol{\Lambda}_{D^{q}}^{\dagger}\right)_{i j}+\frac{6}{5} g_{Y}^{2}\left(\mathbf{Y}_{E} \mathbf{Y}_{E}^{\dagger}+\boldsymbol{\Lambda}_{E^{q}} \boldsymbol{\Lambda}_{E^{q}}^{\dagger}\right)_{i j}, \\
\left(\gamma_{E_{i}}^{(2) E_{j}}\right)_{g-y}= & \left(6 g_{2}^{2}-\frac{6}{5} g_{Y}^{2}\right)\left(\mathbf{Y}_{E}^{\dagger} \mathbf{Y}_{E}\right)_{j i}+\left(3 g_{2}^{2}-\frac{3}{5} g_{Y}^{2}\right) \operatorname{Tr}\left(\boldsymbol{\Lambda}_{E^{i}} \boldsymbol{\Lambda}_{E^{j}}^{\dagger}\right), \\
\left(\gamma_{Q_{i}}^{(2) Q_{j}}\right)_{g-y}= & \frac{2}{5} g_{Y}^{2}\left[\left(\mathbf{Y}_{D} \mathbf{Y}_{D}^{\dagger}+2 \mathbf{Y}_{U} \mathbf{Y}_{U}^{\dagger}\right)_{i j}+\left(\boldsymbol{\Lambda}_{D^{q}}^{\dagger} \boldsymbol{\Lambda}_{D^{q}}\right)_{j i}\right], \\
\left(\gamma_{D_{i}}^{(2) D_{j}}\right)_{g-y}= & \left(\frac{16}{3} g_{3}^{2}+\frac{16}{15} g_{Y}^{2}\right)\left(\boldsymbol{\Lambda}_{U^{q}} \boldsymbol{\Lambda}_{U^{q}}^{\dagger}\right)_{i j} \\
& +\left(6 g_{2}^{2}+\frac{2}{5} g_{Y}^{2}\right)\left[\left(\mathbf{Y}_{D} \mathbf{Y}_{D}^{\dagger}\right)_{i j}+\operatorname{Tr}\left(\boldsymbol{\Lambda}_{D^{i}} \boldsymbol{\Lambda}_{D^{j}}^{\dagger}\right)\right], \\
\left(\gamma_{U_{i}}^{(2) U_{j}}\right)_{g-y}= & \left(6 g_{2}^{2}-\frac{2}{5} g_{Y}^{2}\right)\left(\mathbf{Y}_{U} \mathbf{Y}_{U}^{\dagger}\right)_{i j}+\left(\frac{8}{3} g_{3}^{2}-\frac{4}{15} g_{Y}^{2}\right) \operatorname{Tr}\left(\boldsymbol{\Lambda}_{U^{i}} \boldsymbol{\Lambda}_{U^{j}}^{\dagger}\right), \\
\left(\gamma_{H_{1}}^{(2) H_{1}}\right)_{g-y}= & \left(16 g_{3}^{2}-\frac{2}{5} g_{Y}^{2}\right) \operatorname{Tr}\left(\mathbf{Y}_{D} \mathbf{Y}_{D}^{\dagger}\right)+\frac{6}{5} g_{Y}^{2} \operatorname{Tr}\left(\mathbf{Y}_{E} \mathbf{Y}_{E}^{\dagger}\right), \\
\left(\gamma_{H_{2}}^{(2) H_{2}}\right)_{g-y}= & \left(16 g_{3}^{2}+\frac{4}{5} g_{Y}^{2}\right) \operatorname{Tr}\left(\mathbf{Y}_{U} \mathbf{Y}_{U}^{\dagger}\right), \\
\left(\gamma_{L_{i}}^{(2) H_{1}}\right)_{g-y}= & \left(16 g_{3}^{2}-\frac{2}{5} g_{Y}^{2}\right)\left(\boldsymbol{\Lambda}_{D^{q}} \mathbf{Y}_{D}^{*}\right)_{i q}+\frac{6}{5} g_{Y}^{2}\left(\boldsymbol{\Lambda}_{E^{q}} \mathbf{Y}_{E}^{*}\right)_{i q} .
\end{aligned}
$$

The pure Yukawa two-loop anomalous dimensions are given by

$$
\begin{aligned}
-\left(\gamma_{L_{i}}^{(2) L_{j}}\right)_{\text {yukawa }} & =2\left(\mathbf{Y}_{E} \mathbf{Y}_{E}^{\dagger} \mathbf{Y}_{E} \mathbf{Y}_{E}^{\dagger}\right)_{i j}+\left(\mathbf{Y}_{E} \mathbf{Y}_{E}^{\dagger}\right)_{i j} \operatorname{Tr}\left(\mathbf{Y}_{E} \mathbf{Y}_{E}^{\dagger}+3 \mathbf{Y}_{D} \mathbf{Y}_{D}^{\dagger}\right) \\
& +\left(\mathbf{Y}_{E}\right)_{i n}\left(\mathbf{Y}_{E}^{\dagger}\right)_{r j} \operatorname{Tr}\left(\boldsymbol{\Lambda}_{E^{n}}^{\dagger} \boldsymbol{\Lambda}_{E^{r}}\right)+3\left(\mathbf{Y}_{E}\right)_{i m}\left(\boldsymbol{\Lambda}_{E^{m}}^{\dagger} \boldsymbol{\Lambda}_{D^{q}} \mathbf{Y}_{D}^{*}\right)_{j q} \\
& +\left(\mathbf{Y}_{E}\right)_{i m}\left(\mathbf{Y}_{E}^{\dagger} \boldsymbol{\Lambda}_{E^{q}} \boldsymbol{\Lambda}_{E^{m}}^{*}\right)_{q j}+\left(\boldsymbol{\Lambda}_{E^{n}} \boldsymbol{\Lambda}_{E^{r}}^{\dagger}\right)_{i j} \operatorname{Tr}\left(\boldsymbol{\Lambda}_{E^{n}}^{\dagger} \boldsymbol{\Lambda}_{E^{r}}\right)
\end{aligned}
$$




$$
\begin{aligned}
& +2\left(\boldsymbol{\Lambda}_{E^{n}} \boldsymbol{\Lambda}_{E^{r}}^{*}\right)_{i j}\left(\mathbf{Y}_{E}^{\dagger} \mathbf{Y}_{E}\right)_{n r}+\left(\boldsymbol{\Lambda}_{E^{m}} \mathbf{Y}_{E}^{*} \mathbf{Y}_{E} \boldsymbol{\Lambda}_{E^{m}}^{*}\right)_{i j}+\left(\boldsymbol{\Lambda}_{E^{m}} \boldsymbol{\Lambda}_{E^{q}}^{*} \mathbf{Y}_{E}\right)_{i q}\left(\mathbf{Y}_{E}^{\dagger}\right)_{m j} \\
& +3\left(\boldsymbol{\Lambda}_{E^{m}} \boldsymbol{\Lambda}_{D^{q}}^{*} \mathbf{Y}_{D}\right)_{i q}\left(\mathbf{Y}_{E}^{\dagger}\right)_{m j}+3\left(\boldsymbol{\Lambda}_{E^{m}} \boldsymbol{\Lambda}_{D^{q}}^{*} \boldsymbol{\Lambda}_{D^{q}}^{T} \boldsymbol{\Lambda}_{E^{m}}^{*}\right)_{i j} \\
& +6\left(\boldsymbol{\Lambda}_{D^{n}} \boldsymbol{\Lambda}_{D^{r}}^{\dagger}\right)_{i j}\left(\left(\mathbf{Y}_{D}^{\dagger} \mathbf{Y}_{D}\right)_{n r}+\left(\boldsymbol{\Lambda}_{U^{q}}^{*} \boldsymbol{\Lambda}_{U^{q}}\right)_{n r}+\operatorname{Tr}\left(\boldsymbol{\Lambda}_{D^{n}}^{\dagger} \boldsymbol{\Lambda}_{D^{r}}\right)\right) \\
& +3\left(\boldsymbol{\Lambda}_{D^{m}} \mathbf{Y}_{D}^{*} \mathbf{Y}_{D}^{T} \boldsymbol{\Lambda}_{D^{m}}^{\dagger}\right)_{i j}+3\left(\boldsymbol{\Lambda}_{D^{m}} \mathbf{Y}_{U}^{*} \mathbf{Y}_{U}^{T} \boldsymbol{\Lambda}_{D^{m}}^{\dagger}\right)_{i j}+\left(\boldsymbol{\Lambda}_{E^{m}} \boldsymbol{\Lambda}_{E^{q}}^{*} \boldsymbol{\Lambda}_{E^{q}} \boldsymbol{\Lambda}_{E^{m}}^{*}\right)_{i j} \\
& +3\left(\boldsymbol{\Lambda}_{D^{m}} \boldsymbol{\Lambda}_{D^{q}}^{\dagger} \boldsymbol{\Lambda}_{D^{q}} \boldsymbol{\Lambda}_{D^{m}}^{\dagger}\right)_{i j}, \\
& -\left(\gamma_{E_{i}}^{(2) E_{j}}\right)_{\text {yukawa }}=2\left(\mathbf{Y}_{E}^{\dagger} \mathbf{Y}_{E}\right)_{j i} \operatorname{Tr}\left(\mathbf{Y}_{E}^{\dagger} \mathbf{Y}_{E}+3 \mathbf{Y}_{D} \mathbf{Y}_{D}^{\dagger}\right)+2\left(\mathbf{Y}_{E}^{\dagger} \mathbf{Y}_{E} \mathbf{Y}_{E}^{\dagger} \mathbf{Y}_{E}\right)_{j i} \\
& +2\left(\mathbf{Y}_{E}^{\dagger} \boldsymbol{\Lambda}_{E^{q}} \boldsymbol{\Lambda}_{E^{j}}^{\dagger} \mathbf{Y}_{E}\right)_{q i}+6\left(\mathbf{Y}_{D}^{\dagger} \boldsymbol{\Lambda}_{D^{q}}^{T} \boldsymbol{\Lambda}_{E^{j}}^{\dagger} \mathbf{Y}_{E}\right)_{q i}+2\left(\mathbf{Y}_{E}^{\dagger} \boldsymbol{\Lambda}_{E^{q}}^{T} \boldsymbol{\Lambda}_{E^{q}}^{\dagger} \mathbf{Y}_{E}\right)_{j i} \\
& +6\left(\mathbf{Y}_{E}^{\dagger} \boldsymbol{\Lambda}_{D^{q}} \boldsymbol{\Lambda}_{D^{q}}^{\dagger} \mathbf{Y}_{E}\right)_{j i}+2 \operatorname{Tr}\left(\boldsymbol{\Lambda}_{E^{j}}^{*} \mathbf{Y}_{E} \mathbf{Y}_{E^{\dagger}}^{\dagger} \boldsymbol{\Lambda}_{E^{i}}^{T}\right)+2\left(\mathbf{Y}_{E}^{\dagger} \boldsymbol{\Lambda}_{E^{i}} \boldsymbol{\Lambda}_{E^{q}}^{*} \mathbf{Y}_{E}\right)_{j q} \\
& +6\left(\mathbf{Y}_{E}^{\dagger} \boldsymbol{\Lambda}_{E^{i}} \boldsymbol{\Lambda}_{D^{q}}^{*} \mathbf{Y}_{D}\right)_{j q}+2 \operatorname{Tr}\left(\boldsymbol{\Lambda}_{E^{i}} \boldsymbol{\Lambda}_{E^{q}}^{*} \boldsymbol{\Lambda}_{E^{q}} \boldsymbol{\Lambda}_{E^{j}}^{\dagger}\right)+6 \operatorname{Tr}\left(\boldsymbol{\Lambda}_{E^{j}}^{*} \boldsymbol{\Lambda}_{D^{q}} \boldsymbol{\Lambda}_{D^{q}}^{\dagger} \boldsymbol{\Lambda}_{E^{i}}^{T}\right), \\
& -\left(\gamma_{Q_{i}}^{(2) Q_{j}}\right)_{\text {yukawa }}=2\left(\mathbf{Y}_{D} \mathbf{Y}_{D}^{\dagger} \mathbf{Y}_{D} \mathbf{Y}_{D}^{\dagger}\right)_{i j}+\left(\mathbf{Y}_{D} \mathbf{Y}_{D}^{\dagger}\right)_{i j} \operatorname{Tr}\left(\mathbf{Y}_{E}^{\dagger} \mathbf{Y}_{E}+3 \mathbf{Y}_{D}^{\dagger} \mathbf{Y}_{D}\right) \\
& +2\left(\mathbf{Y}_{U} \mathbf{Y}_{U}^{\dagger} \mathbf{Y}_{U} \mathbf{Y}_{U}^{\dagger}\right)_{i j}+3\left(\mathbf{Y}_{U} \mathbf{Y}_{U}^{\dagger}\right)_{i j} \operatorname{Tr}\left(\mathbf{Y}_{U}^{\dagger} \mathbf{Y}_{U}\right) \\
& +2\left(\mathbf{Y}_{D} \boldsymbol{\Lambda}_{U^{q}}^{*} \boldsymbol{\Lambda}_{U^{q}} \mathbf{Y}_{D}^{\dagger}\right)_{i j}+3\left(\boldsymbol{\Lambda}_{D^{m}}^{\dagger} \boldsymbol{\Lambda}_{D^{q}} \boldsymbol{\Lambda}_{D^{q}}^{\dagger} \boldsymbol{\Lambda}_{D^{m}}\right)_{j i} \\
& +2\left(\mathbf{Y}_{D}\right)_{i n}\left(\mathbf{Y}_{D}^{\dagger}\right)_{r j} \operatorname{Tr}\left(\boldsymbol{\Lambda}_{D^{n}}^{\dagger} \boldsymbol{\Lambda}_{D^{r}}\right)+\left(\boldsymbol{\Lambda}_{D^{m}}^{\dagger} \mathbf{Y}_{E} \mathbf{Y}_{E}^{\dagger} \boldsymbol{\Lambda}_{D^{m}}\right)_{j i} \\
& +\left(\boldsymbol{\Lambda}_{D^{m}}^{\dagger} \boldsymbol{\Lambda}_{E^{q}}^{T} \boldsymbol{\Lambda}_{E^{q}}^{\dagger} \boldsymbol{\Lambda}_{D^{m}}\right)_{j i}+\left(\mathbf{Y}_{D}\right)_{i m}\left(3 \mathbf{Y}_{D^{\dagger}}^{\dagger} \boldsymbol{\Lambda}_{D^{q}}^{T} \boldsymbol{\Lambda}_{D^{m}}^{*}+\mathbf{Y}_{E}^{\dagger} \boldsymbol{\Lambda}_{E^{q}} \boldsymbol{\Lambda}_{D^{m}}^{*}\right)_{q j} \\
& +\left(\mathbf{Y}_{U}\right)_{i n}\left(\mathbf{Y}_{U}^{\dagger}\right)_{r j} \operatorname{Tr}\left(\boldsymbol{\Lambda}_{U^{n}}^{\dagger} \boldsymbol{\Lambda}_{U^{r}}\right) \\
& +2\left(\boldsymbol{\Lambda}_{D^{r}}^{\dagger} \boldsymbol{\Lambda}_{D^{n}}\right)_{j i}\left(\left(\mathbf{Y}_{D}^{\dagger} \mathbf{Y}_{D}+\boldsymbol{\Lambda}_{U^{q}}^{*} \boldsymbol{\Lambda}_{U^{q}}\right)_{n r}+\operatorname{Tr}\left(\boldsymbol{\Lambda}_{D^{n}}^{\dagger} \boldsymbol{\Lambda}_{D^{r}}\right)\right) \\
& +\left(\mathbf{Y}_{D}^{\dagger}\right)_{m j}\left(3 \boldsymbol{\Lambda}_{D^{m}}^{T} \boldsymbol{\Lambda}_{D^{q}}^{*} \mathbf{Y}_{D}+\boldsymbol{\Lambda}_{D^{m}}^{T} \boldsymbol{\Lambda}_{E^{q}}^{*} \mathbf{Y}_{E}\right)_{i q}, \\
& -\left(\gamma_{D_{i}}^{(2) D_{j}}\right)_{\text {yukawa }}=2\left(\mathbf{Y}_{D}^{\dagger} \mathbf{Y}_{D} \mathbf{Y}_{D}^{\dagger} \mathbf{Y}_{D}\right)_{j i}+2\left(\mathbf{Y}_{D}^{\dagger} \mathbf{Y}_{U} \mathbf{Y}_{U}^{\dagger} \mathbf{Y}_{D}\right)_{j i} \\
& +2\left(\mathbf{Y}_{D}^{\dagger} \mathbf{Y}_{D}\right)_{j i} \operatorname{Tr}\left(\mathbf{Y}_{E}^{\dagger} \mathbf{Y}_{E}+3 \mathbf{Y}_{D}^{\dagger} \mathbf{Y}_{D}\right)+2\left(\mathbf{Y}_{D}^{\dagger} \boldsymbol{\Lambda}_{D^{q}}^{T} \boldsymbol{\Lambda}_{D^{q}}^{*} \mathbf{Y}_{D}\right)_{j i} \\
& +2\left(\mathbf{Y}_{E}^{\dagger} \boldsymbol{\Lambda}_{E^{q}} \boldsymbol{\Lambda}_{D^{j}}^{*} \mathbf{Y}_{D}\right)_{q i}+6\left(\mathbf{Y}_{D}^{\dagger} \boldsymbol{\Lambda}_{D^{q}}^{T} \boldsymbol{\Lambda}_{D^{j}}^{*} \mathbf{Y}_{D}\right)_{q i} \\
& +2 \operatorname{Tr}\left(\boldsymbol{\Lambda}_{D^{i}}^{T} \boldsymbol{\Lambda}_{D^{j}}^{*}\left(\mathbf{Y}_{D} \mathbf{Y}_{D}^{\dagger}+\mathbf{Y}_{U} \mathbf{Y}_{U}^{\dagger}\right)+\boldsymbol{\Lambda}_{D^{i}} \boldsymbol{\Lambda}_{D^{q}}^{\dagger} \boldsymbol{\Lambda}_{D^{q}} \boldsymbol{\Lambda}_{D^{j}}^{\dagger}\right) \\
& +\operatorname{Tr}\left(6 \boldsymbol{\Lambda}_{D^{q}} \boldsymbol{\Lambda}_{D^{q}}^{\dagger} \boldsymbol{\Lambda}_{D^{i}} \boldsymbol{\Lambda}_{D^{j}}^{\dagger}+2 \boldsymbol{\Lambda}_{D^{i}} \boldsymbol{\Lambda}_{D^{j}}^{\dagger} \boldsymbol{\Lambda}_{E^{q}}^{T} \boldsymbol{\Lambda}_{E^{q}}^{\dagger}+2 \mathbf{Y}_{E} \mathbf{Y}_{E}^{\dagger} \boldsymbol{\Lambda}_{D^{i}} \boldsymbol{\Lambda}_{D^{j}}^{\dagger}\right) \\
& +2\left(\mathbf{Y}_{D}^{\dagger} \boldsymbol{\Lambda}_{D^{i}}^{T} \boldsymbol{\Lambda}_{E q}^{*} \mathbf{Y}_{E}\right)_{j q}+6\left(\mathbf{Y}_{D^{\dagger}}^{\dagger} \boldsymbol{\Lambda}_{D^{i}}^{T} \boldsymbol{\Lambda}_{D^{q}}^{*} \mathbf{Y}_{D}\right)_{j q} \\
& +4\left(\boldsymbol{\Lambda}_{U^{m}}\right)_{i n}\left(\boldsymbol{\Lambda}_{U^{m}}^{\dagger}\right)_{j r} \operatorname{Tr}\left(\boldsymbol{\Lambda}_{D^{n}}^{\dagger} \boldsymbol{\Lambda}_{D^{r}}\right) \\
& +4\left(\boldsymbol{\Lambda}_{U^{m}}\left(\mathbf{Y}_{D}^{\dagger} \mathbf{Y}_{D}+\boldsymbol{\Lambda}_{U^{q}}^{*} \boldsymbol{\Lambda}_{U^{q}}\right) \boldsymbol{\Lambda}_{U^{m}}^{*}\right)_{i j} \\
& +2\left(\boldsymbol{\Lambda}_{U^{n}} \boldsymbol{\Lambda}_{U^{r}}^{*}\right)_{i j}\left(2\left(\mathbf{Y}_{U}^{\dagger} \mathbf{Y}_{U}\right)_{n r}+\operatorname{Tr}\left(\boldsymbol{\Lambda}_{U^{n}}^{\dagger} \boldsymbol{\Lambda}_{U^{r}}\right)\right) \text {, } \\
& -\left(\gamma_{U_{i}}^{(2) U_{j}}\right)_{\text {yukawa }}=2\left(\mathbf{Y}_{U}^{\dagger} \mathbf{Y}_{U} \mathbf{Y}_{U}^{\dagger} \mathbf{Y}_{U}\right)_{j i}+2\left(\mathbf{Y}_{U}^{\dagger} \mathbf{Y}_{D} \mathbf{Y}_{D}^{\dagger} \mathbf{Y}_{U}\right)_{j i}+6\left(\mathbf{Y}_{U}^{\dagger} \mathbf{Y}_{U}\right)_{j i} \operatorname{Tr}\left(\mathbf{Y}_{U} \mathbf{Y}_{U}^{\dagger}\right) \\
& +2\left(\mathbf{Y}_{U}^{T} \boldsymbol{\Lambda}_{D^{q}}^{\dagger} \boldsymbol{\Lambda}_{D^{q}} \mathbf{Y}_{U}^{*}\right)_{i j}+4 \operatorname{Tr}\left(\boldsymbol{\Lambda}_{U^{j}}^{\dagger} \boldsymbol{\Lambda}_{U^{i}} \mathbf{Y}_{D}^{\dagger} \mathbf{Y}_{D}\right)
\end{aligned}
$$




$$
\begin{aligned}
& +4 \operatorname{Tr}\left(\boldsymbol{\Lambda}_{U^{j}}^{\dagger} \boldsymbol{\Lambda}_{U^{i}} \boldsymbol{\Lambda}_{U^{q}}^{*} \boldsymbol{\Lambda}_{U^{q}}\right)+4\left(\boldsymbol{\Lambda}_{U^{j}}^{\dagger} \boldsymbol{\Lambda}_{U^{i}}\right)_{r n} \operatorname{Tr}\left(\boldsymbol{\Lambda}_{D^{n}}^{\dagger} \boldsymbol{\Lambda}_{D^{r}}\right), \\
& -\left(\gamma_{H_{1}}^{(2) H_{1}}\right)_{\text {yukawa }}=\operatorname{Tr}\left(3 \mathbf{Y}_{E} \mathbf{Y}_{E}^{\dagger} \mathbf{Y}_{E} \mathbf{Y}_{E}^{\dagger}+9 \mathbf{Y}_{D}^{\dagger} \mathbf{Y}_{D} \mathbf{Y}_{D}^{\dagger} \mathbf{Y}_{D}+3 \mathbf{Y}_{D} \mathbf{Y}_{D}^{\dagger} \mathbf{Y}_{U} \mathbf{Y}_{U}^{\dagger}\right) \\
& +\operatorname{Tr}\left(3 \boldsymbol{\Lambda}_{D^{q}} \boldsymbol{\Lambda}_{D^{q}}^{\dagger} \mathbf{Y}_{E} \mathbf{Y}_{E}^{\dagger}-\boldsymbol{\Lambda}_{E^{q}} \boldsymbol{\Lambda}_{E^{q}}^{\dagger} \mathbf{Y}_{E} \mathbf{Y}_{E}^{\dagger}-6 \mathbf{Y}_{D}^{\dagger} \mathbf{Y}_{D} \boldsymbol{\Lambda}_{U^{q}}^{\dagger} \boldsymbol{\Lambda}_{U^{q}}\right. \\
& \left.+3 \mathbf{Y}_{D} \mathbf{Y}_{D}^{\dagger} \boldsymbol{\Lambda}_{D^{q}}^{\dagger} \boldsymbol{\Lambda}_{D^{q}}\right)+\left(\mathbf{Y}_{E}^{\dagger} \mathbf{Y}_{E}\right)_{r n} \operatorname{Tr}\left(\boldsymbol{\Lambda}_{E^{n}}^{\dagger} \boldsymbol{\Lambda}_{E^{r}}\right) \\
& +6\left(\mathbf{Y}_{D}^{\dagger} \mathbf{Y}_{D}\right)_{r n} \operatorname{Tr}\left(\boldsymbol{\Lambda}_{D^{n}}^{\dagger} \boldsymbol{\Lambda}_{D^{r}}\right) \\
& -\left(\gamma_{\bar{H}}^{(2) \bar{H}}\right)_{\text {yukawa }}=\operatorname{Tr}\left(9 \mathbf{Y}_{U} \mathbf{Y}_{U}^{\dagger} \mathbf{Y}_{U} \mathbf{Y}_{U}^{\dagger}+3 \mathbf{Y}_{U} \mathbf{Y}_{U}^{\dagger} \mathbf{Y}_{D} \mathbf{Y}_{D}^{\dagger}+3 \mathbf{Y}_{U} \mathbf{Y}_{U}^{\dagger} \Lambda_{D^{q}}^{T} \boldsymbol{\Lambda}_{D^{q}}^{*}\right) \\
& +3\left(\mathbf{Y}_{U}^{\dagger} \mathbf{Y}_{U}\right)_{r n} \operatorname{Tr}\left(\boldsymbol{\Lambda}_{U^{n}}^{\dagger} \boldsymbol{\Lambda}_{U^{r}}\right) \text {, } \\
& -\left(\gamma_{L_{i}}^{(2) H_{1}}\right)_{\text {yukawa }}=3\left(\mathbf{Y}_{E} \mathbf{Y}_{E}^{\dagger} \boldsymbol{\Lambda}_{D^{q}} \mathbf{Y}_{D}^{*}\right)_{i q}+\left(\mathbf{Y}_{E} \mathbf{Y}_{E}^{\dagger} \boldsymbol{\Lambda}_{E^{q}}^{T} \mathbf{Y}_{E}^{*}\right)_{i q} \\
& +3\left(\mathbf{Y}_{E}^{\dagger} \mathbf{Y}_{E} \mathbf{Y}_{E}^{\dagger} \boldsymbol{\Lambda}_{E^{n}}^{T}\right)_{n i}+\left(\boldsymbol{\Lambda}_{E^{n}} \mathbf{Y}_{E}^{*}\right)_{i r} \operatorname{Tr}\left(\boldsymbol{\Lambda}_{E^{n}}^{\dagger} \boldsymbol{\Lambda}_{E^{r}}\right) \\
& +\left(\boldsymbol{\Lambda}_{E^{m}} \boldsymbol{\Lambda}_{E^{q}}^{*} \boldsymbol{\Lambda}_{E^{q}} \mathbf{Y}_{E}^{*}\right)_{i m}+3\left(\mathbf{Y}_{E}^{\dagger} \boldsymbol{\Lambda}_{D^{q}} \boldsymbol{\Lambda}_{D^{q}}^{\dagger} \boldsymbol{\Lambda}_{E^{m}}^{T}\right)_{m i} \\
& +9\left(\mathbf{Y}_{D}^{\dagger} \mathbf{Y}_{D} \mathbf{Y}_{D}^{\dagger} \boldsymbol{\Lambda}_{D^{n}}^{T}\right)_{n i}+6\left(\mathbf{Y}_{D}^{\dagger} \boldsymbol{\Lambda}_{D^{n}}^{T}\right)_{r i} \operatorname{Tr}\left(\boldsymbol{\Lambda}_{D^{n}}^{\dagger} \boldsymbol{\Lambda}_{D^{r}}\right) \\
& +6\left(\boldsymbol{\Lambda}_{U^{q}}^{*} \boldsymbol{\Lambda}_{U^{q}} \mathbf{Y}_{D}^{\dagger} \boldsymbol{\Lambda}_{D^{n}}^{T}\right)_{n i}+3\left(\mathbf{Y}_{D}^{\dagger} \mathbf{Y}_{U} \mathbf{Y}_{U^{\dagger}}^{\dagger} \boldsymbol{\Lambda}_{D^{m}}^{T}\right)_{m i} \\
& +3\left(\boldsymbol{\Lambda}_{D^{m}} \boldsymbol{\Lambda}_{D^{q}}^{\dagger} \boldsymbol{\Lambda}_{D^{q}} \mathbf{Y}_{D}^{*}\right)_{i m} .
\end{aligned}
$$

This completes the renormalization group equations for the Yukawa couplings at two-loop. Before we discuss applications we briefly consider the renormalization of the bilinear terms.

\subsection{Bi-Linear Terms}

Following the general equations given in MV the renormalization group equations for the bilinear terms now including all R-parity violating effects are given by

$$
\begin{aligned}
\frac{d}{d t} \mu & =\mu\left\{\Gamma_{H_{1}}^{H_{1}}+\Gamma_{H_{2}}^{H_{2}}\right\}+\kappa^{i} \Gamma_{L_{i}}^{H_{1}}, \\
\frac{d}{d t} \kappa^{i} & =\kappa^{i} \Gamma_{H_{2}}^{H_{2}}+\kappa^{p} \Gamma_{L_{p}}^{L_{i}}+\mu \Gamma_{H_{1}}^{L_{i}} .
\end{aligned}
$$

The anomalous dimensions at two-loop are given in the previous subsections. As already noted in MV the bi-linear terms do not appear in the equations for the Yukawa couplings.

\subsection{Discussion}

Equation (2.53) implies that for $\kappa_{i}=0$ at tree-level for all $i$, a non-zero $\kappa_{i}$ is generated via the $\mu$-term. However, as is well known, if the coefficient of the corresponding soft breaking term equals that of the superpotential term, then the terms $\kappa_{i} L_{i} H_{1}$ in the superpotential can be rotated away through a redefinition of the $L_{i}$ and $H_{1}$ [19]. If we are considering the one-loop or two-loop renormalized Lagrangian then we must make this rotation after renormalization. Again, there will be no term $\kappa_{i} L_{i} H_{1}$ in the Lagrangian, the rotation matrix will of course differ. Thus there is no mixing between $L_{i}$ and $H_{1}$ and $\Gamma_{L_{i}}^{H_{1}}=0$ is guaranteed by 
the relevant counterterms at two-loop. This then also applies to the Eqs(2.13, 2.14, 2.16,2.17) in Section 2.2.1 and Eq.(2.53) in Section 2.3. If the Lagrangian has additional symmetries which distingiush between $L_{i}$ and $H_{1}$ and allows $L_{i} H_{2}$ in $W_{\mathbb{R}_{p}}$ then these terms must be retained.

The two-loop renormalization group equations for the Yukawa couplings also respect several symmetries. If at some scale for example $\lambda_{i j k}^{\prime \prime}=0$ for all $i, j, k$ then baryon parity, $B_{p}$, is conserved at this scale. There are no $\not B_{p}$-couplings in the theory and thus in perturbation theory no $\not B_{p}$-couplings are generated, i.e. the RGEs preserve $\lambda_{i j k}^{\prime \prime}=0$. Analogously lepton parity, once imposed, is also preserved by the RGEs. If at some scale $\lambda_{i j k}=\lambda_{i j k}^{\prime \prime}=0$ for all $i, j, k$ and only one lepton flavour is violated, e.g. $\lambda_{3 j k}^{\prime} \neq 0$ then this is also true for all scales, provided $\Gamma_{L_{n}}^{H_{1}}=0$. So the lepton number violating couplings $L Q \bar{D}$ and $L L \bar{E}$ decouple in this limit. If the neutrino masses are non-zero then this is no longer true. The electron

mass matrix $\mathbf{Y}_{E}$ then contains off-diagonal entries which generate off diagonal $\Gamma_{E_{j}}^{E_{i}}, \Gamma_{L_{j}}^{L_{i}}$ via the RGEs. But the effects will be very small and can thus be neglected in most circumstances. However, if we assume only $\lambda_{111}^{\prime} \neq 0$ at some scale then through the quark CKM-mixing the other terms $\lambda_{1 i j}^{\prime}$ will be generated.

Our results agree with MV for the MSSM Yukawa couplings. We also agree with the one-loop results 13, 12, 14.

\section{Unification}

We now apply our two-loop RG-equations to the questions of unification. We shall assume as a first approximation that the $R_{p}$-couplings have a similar hierarchy to the SM Yukawa couplings and thus only consider one coupling at a time. The third generation couplings have the weakest bounds (1.4) and can thus lead to the largest effects. We shall consider the three cases

$$
\text { LLE }: \lambda_{323}, \quad \mathbf{L Q} \overline{\mathbf{D}}: \lambda_{333}^{\prime}, \quad \overline{\mathbf{U}} \overline{\mathbf{D}} \overline{\mathrm{D}}: \lambda_{323}^{\prime \prime} \text {. }
$$

We assume that in each case the respective operator decouples from the other $\not R_{p}$-operators whose couplings we set to zero. Is this approximation consistent, i.e. is it stable under the RGEs? First consider the $L L \bar{E}$ operator. The coupling $\lambda_{323}$ only violates $L_{\mu}$ and could thus also generate non-zero $\lambda_{121}$. However, $\lambda_{121}$ decouples from $\lambda_{323}$ if it is the only non-vanishing operator at some scale. The other $L L \bar{E}$ operators are protected by global $L_{e}$ and $L_{\tau}$. It is thus consistent to only consider a non-zero $\lambda_{323}$.

The coupling $\lambda_{333}^{\prime}$ will generate $\lambda_{3 i j}^{\prime} \neq 0$ in higher order. However, these terms are proportional to third generation off-diagonal CKM-matrix elements which are very small and can be safely ignored. Thus for $\lambda_{323}^{\prime}$ the decoupling assumption is also good. For $\lambda_{323}^{\prime \prime}$ the higher order mixing with the couplings $\lambda_{i 2 k}^{\prime \prime}$ is also very small. The mixing with $\lambda_{313}^{\prime \prime}$ is Cabbibo suppressed and involves a first generation Higgs Yukawa coupling and can be neglected. Thus in all three cases the decoupling is a good assumption. In line with this argument we assume 
the following form for the Higgs-Yukawa matrices

$$
\mathbf{Y}_{E}=\operatorname{diag}\left(0,0, \lambda_{\tau}\right), \quad \mathbf{Y}_{D}=\operatorname{diag}\left(0,0, \lambda_{b}\right), \quad \mathbf{Y}_{U}=\operatorname{diag}\left(0,0, \lambda_{t}\right)
$$

In order to determine the scale of unification we numerically solve the renormalization group equations. Our analysis is analogous to that of Refs [31] to which we refer for details. We also do not consider GUT threshold corrections. Our analysis differs in that we restrict ourselves to three generations but instead add in turn one of the three $R_{p}$-Yukawa couplings (3.1). Thus we run the full set of equations including the two-loop correction of the Yukawa couplings to the running of the gauge couplings (2.8). We use the experimental values $\alpha_{e m}^{-1}\left(M_{Z}\right)=127.9$ and $\sin ^{2} \theta_{W}\left(M_{Z}\right)=0.2324$ [2]. We consider only a single supersymmetry mass scale $M_{S U S Y}=M_{Z}$. For more realistic spectra $M_{S U S Y}$ can be considered an effective mass scale which enters in the RGEs [2, 3]. The supersymmetric mass spectrum can be highly non-uniform with the masses typically larger than $M_{S U S Y}$. We then iteratively determine the value of $\alpha_{3}\left(M_{Z}\right)$ and thus also the unification mass scale $M_{U}$ and the coupling at the unification scale $\alpha_{U}$ [31]. We have chosen a running top mass of $m_{t}\left(m_{t}\right)=165 \mathrm{GeV}$ which corresponds to a pole mass $m_{t}=175 \mathrm{GeV}$ in agreement with the discovery at the Tevatron [24]. We fix the running bottom quark mass at $m_{b}\left(m_{b}\right)=4.25 \mathrm{GeV}$ [32].

We solve the RGEs for different values of the $\not R_{p}$-coupling at the weak scale, starting from zero. The maximal value we consider is where the running coupling reaches the perturbative limit at the unification scale. So we require

$$
\frac{\lambda_{R_{p}}^{2}\left(M_{U}\right)}{4 \pi}<1
$$

or $\lambda_{\not R_{p}}\left(M_{U}\right)<3.5$.

One of the encouraging aspects of supersymmetric grand unified theories is the possibility of bottom-tau unification at $M_{U}$. Similar to gauge coupling unification this is not possible in standard GUTs. There has been a large interest in the literature [22, 23, 1, 3, 33] in the restrictions on the unification scenario from bottom-tau unification. Requiring bottom-tau unification leads to a strict relation between the running top quark mass and $\tan \beta$. For the experimental value of $m_{t}$ [24], $\tan \beta$ is predicted to be very close to 1.5 or around 60 [34]. We are interested in how the effects of $\not R_{p}$ can relax this strict relation and allow a larger range of $\tan \beta$. As a model scenario we consider $\tan \beta=5$ which is well away from the solutions in the MSSM. We then investigate the possibilities for a top fix-point solution as well as bottom-tau unification including the $\not R_{p}$-effects. We do not consider the other GUT mass ratios.

\subsection{Gauge Coupling Unification}

Before determining the effects of $\lambda_{R_{p}} \neq 0$ we would also like to consider the effect from the non-vanishing top quark Yukawa coupling. Thus we first determine the unification values for $\lambda_{t}=0$ and $\lambda_{\not R_{p}}=0$. We obtain

$$
\alpha_{3}\left(M_{Z}\right)=0.128, \quad M_{U}=2.9810^{16} \mathrm{GeV}, \quad \alpha_{U}=0.043 .
$$


In order to discuss the effects of the non-zero Yukawa couplings we consider the unification parameters as functions of the weak scale values of $\lambda_{t}$ and $\lambda_{\mathbb{R}_{p}}$

$$
\alpha_{s}\left(M_{Z}, \lambda_{t}, \lambda_{\not R_{p}}\right), \quad M_{U}\left(\lambda_{t}, \lambda_{\not R_{p}}\right), \quad \alpha_{U}\left(\lambda_{t}, \lambda_{\not R_{p}}\right) .
$$

and define the ratios

$$
\begin{aligned}
R_{\alpha_{3}}\left(\lambda_{t}, \lambda_{\not R_{p}}\right) & =\frac{\alpha_{3}\left(M_{Z}, \lambda_{t}, \lambda_{\not R_{p}}\right)}{\alpha_{3}\left(M_{Z}, 0,0\right)}, \\
R_{M_{U}}\left(\lambda_{t}, \lambda_{\not R_{p}}\right) & =\frac{M_{U}\left(\lambda_{t}, \lambda_{\not R_{p}}\right)}{M_{U}(0,0)}, \\
R_{\alpha_{U}}\left(\lambda_{t}, \lambda_{\not R_{p}}\right) & =\frac{\alpha_{U}\left(\lambda_{t}, \lambda_{\not R_{p}}\right)}{\alpha_{U}(0,0)} .
\end{aligned}
$$

We first consider $\lambda_{R_{p}}=0$ and fix $\lambda_{t}\left(M_{Z}\right)=0.44$ so that $m_{t}\left(m_{t}\right)=165 \mathrm{GeV}(\tan \beta=5)$. This shifts the ratios (3.6) as is shown in Figs $1 \mathrm{a}, 2 \mathrm{a}$, and $3 \mathrm{a}, R_{i}\left(\lambda_{t}, 0\right) \neq 1$. It can be read off for $\left(\Lambda_{E^{3}}\right)_{23}\left(M_{Z}\right)=0,\left(\Lambda_{D^{3}}\right)_{33}\left(M_{Z}\right)=0$, or $\left(\Lambda_{U^{3}}\right)_{23}\left(M_{Z}\right)=0$ respectively. The shift is of course identical in the three plots, note however the different scales for $R_{i}$. Due to the large top Yukawa $\alpha_{s}\left(M_{Z}\right)$ and $\alpha_{U}$ are lowered only by about $1 \%$. The unification scale is lowered by about $1.5 \%$.

Next we turn on the $R_{p}$-couplings. In Figures $1 \mathrm{~b}, 2 \mathrm{~b}$, and $3 \mathrm{~b}$ we can read off the value of the $R_{p}$-coupling at the unification scale as a function of the coupling at the weak scale. $\left(\Lambda_{E^{3}}\right)_{23}\left(M_{U}\right)$ reaches its perturbative limit (3.3) for a weak scale value of

$$
\left(\Lambda_{E^{3}}\right)_{23}\left(M_{Z}\right)=0.9
$$

It is worth pointing out that this is the same as the laboratory bound for slepton masses at $1 \mathrm{TeV}$ ! Thus although the laboratory bounds on the $L L \bar{E}$ operators are generally considered to be very strict; for heavy supersymmetric masses they are no stricter than the perturbative limit. At this point $\left(\Lambda_{E^{3}}\right)_{23}\left(M_{U}\right)$ has run off the plot but it should be clear how it extrapolates. All other quantities in Figs. 1a,b are plotted up to $\left(\Lambda_{E^{3}}\right)_{23}\left(M_{Z}\right)=0.9$. We have chosen the scaling of the plot so as to highlight the effects on the other quantities. The perturbative limits for the other couplings are given by

$$
\begin{aligned}
& \left(\Lambda_{D^{3}}\right)_{33}\left(M_{Z}\right)=1.08 \\
& \left(\Lambda_{U^{3}}\right)_{23}\left(M_{Z}\right)=1.14
\end{aligned}
$$

The latter value is about $15 \%$ higher than the 1-loop value previously obtained [13], 14]. This is the same order as the two-loop Yukawa corrections obtained in [1].

In Figures 1a, 2a, 3a we show how the ratios (3.6) change as we turn on the $\not R_{p}$-couplings. For $\left(\Lambda_{E^{3}}\right)_{23}\left(M_{Z}\right), \alpha_{s}$ and $\alpha_{U}$ are practically unchanged except very close to the perturbative limit. However, $M_{U}$ is shifted upwards by up to $10 \%$. For $\left(\Lambda_{E^{3}}\right)_{23}\left(M_{Z}\right) \approx \lambda_{t}\left(M_{Z}\right)$ the decrease due to the top quark is off-set by $\lambda_{R_{p}}$. For $\left(\Lambda_{D^{3}}\right)_{33}\left(M_{Z}\right)$ the maximum combined 
shift in $\alpha_{s}\left(M_{Z}\right)$ is a decrease of $4 \%$ giving a value of $\alpha_{s}\left(M_{Z}\right)=0.123$, in better agreement with the data [35]. $\alpha_{U}$ is decreased slightly. However, $M_{U}$ is decreased by up to $20 \%$. This effect is significantly beyond the effect due to the top quark Yukawa coupling. For $\left(\Lambda_{U^{3}}\right)_{23}\left(M_{Z}\right), \alpha_{U}$ remains practically unchanged. $\alpha_{s}$ now has an overall increase of about $5 \%$ at the perturbative limit corresponding to a value of $\alpha_{s}\left(M_{Z}\right)=0.134$ in disagreement with the experimental value. $M_{U}$ is raised by up to $20 \%$.

Thus we find $\alpha_{U}$ essentially unchanged by $\not R_{p}$-effects. $M_{U}$ can change either way by up to $20 \%$. If we compare this with other effects considered in Ref. [2] we find it of the same order as the uncertainty due to the top quark Yukawa coupling or the effects of possible non-renormalizable operators at beyond the GUT scale. The effect is much smaller than that due to GUT-scale threshold corrections or weak-scale supersymmetric threshold corrections. It is thus much too small an effect to accomodate string unification. The strong coupling can also change either way by up to $5 \%$. A decrease is favoured by the data and is welcome in supersymmetric unification. The effect of the $\not R_{p}$-couplings on $\alpha_{s}\left(M_{Z}\right)$ is of the same order as the effects due to the top-quark Yukawa coupling, GUT-scale threshold effects and high-scale non-renormalizable operators [2].

\section{$3.2 \quad b-\tau$ Unification}

In order to study the unification of the bottom and $\tau$ Yukawa couplings $\lambda_{b}, \lambda_{\tau}$ at $M_{U}$ we define the ratio

$$
R_{b / \tau}\left(M_{U}\right)=\frac{\lambda_{b}\left(M_{U}, \lambda_{\not R_{p}}\right)}{\lambda_{\tau}\left(M_{U}, \lambda_{\not R_{p}}\right)} .
$$

For $\lambda_{R_{p}}=0, \tan \beta=5$ we have

$$
R_{b / \tau}\left(M_{U}\right)=0.74
$$

Thus including the top-quark effects but before turning on the $\not R_{p}$-coupling we are well away from the bottom-tau unification solution $R_{b / \tau}\left(M_{U}\right)=1$. Recall that the uncertainties due to the bottom quark mass are small for small $\tan \beta$. Now we consider the corrections due to the $\not R_{p}$-couplings. The one-loop RGE for $R_{b / \tau}(t)$ is given by

$$
\frac{d R_{b / \tau}(t)}{d t}=R_{b / \tau}(t)\left[\lambda_{t}^{2}+3 \lambda_{b}^{2}-3 \lambda_{\tau}^{2}-3 \lambda_{323}^{2}+2 \lambda_{323}^{\prime \prime 2}+\frac{4}{3} g_{Y}^{2}-\frac{16}{3} g_{3}^{2}\right] .
$$

At one-loop the evolution of $R_{b / \tau}$ is unaffected by the operator $L_{3} Q_{3} \bar{D}_{3}$. The slight rise which we observe in Fig $2 \mathrm{~b}$ is a two-loop effect which is very small. The leading dependence of $R_{b / \tau}$ on $\lambda_{323}$ has a negative sign and as we see in the two-loop result shown in Fig. $1 \mathrm{~b} R_{b / \tau}$ drops significantly. At the perturbative limit it has dropped by a factor of three and $R_{p}$ becomes a dominant effect on the evolution of $R_{b / \tau}$. This is important for the range of $\tan \beta$ which leads to bottom-tau unification. In the MSSM $R_{b / \tau}$ is too large for $\tan \beta \lesssim 1.5$ or $\gtrsim 60$ [34. Including a non-zero operator $\lambda_{323}$ strongly reduces $R_{b / \tau}$ and thus can lead to bottom-tau unification outside the previous regime. 
For $\lambda_{323}^{\prime \prime} \neq 0$ there is an additional positive contribution in the evolution of $R_{b / \tau}(t)$. The full two-loop result shows a clear rise in $R_{b / \tau}(t)$ as a function of $\lambda_{323}^{\prime \prime}$ in Fig. 3b. The maximum increase at the perturbative limit is by $60 \%$. For $\lambda_{323}^{\prime \prime}\left(M_{Z}\right)=1.07$ bottom-tau unification is restored! This is quite remarkable. Even though $\not_{p}$-couplings are usually expected to lead to only small effects they can have a significant impact on our understanding of Yukawaunification. Recall, that grand unification is possible in $\not R_{p}$-theories 19$]$.

From the structure of the couplings it should be clear that for example for $\lambda_{233}^{\prime}$ we get an increase in $R_{b / \tau}\left(M_{U}\right)$ as well since at one-loop it does not couple to $\lambda_{\tau}$. Similarly, $\lambda_{322}^{\prime}$ decreases $R_{b / \tau}$. This leads to further bottom-tau unification points.

\subsection{Fixed Point Structure of the Top Yukawa}

We would next like to discuss the effects of the $\not R_{p}$-couplings on $\lambda_{t}\left(M_{U}\right)$. In particular we are interested in finding fix-point structures. These are defined by zero derivative at $M_{Z}$. For the MSSM parameter-point we have chosen, $\tan \beta=5$, we obtain

$$
\lambda_{t}\left(M_{U}\right)=0.45, \quad \text { for } \lambda_{R_{p}}=0 .
$$

This is indicated in Figs 1b, 2b, and 3b. The one-loop evolution equation of $\lambda_{t}$ is given by

$$
\frac{d \lambda_{t}}{d t}=\lambda_{t}\left[6 \lambda_{t}^{2}+\lambda_{b}^{2}+2 \lambda_{323}^{\prime \prime 2}+\lambda_{333}^{\prime 2}-\left(\frac{13}{15} g_{Y}^{2}+3 g_{2}^{2}+\frac{16}{3} g_{3}^{2}\right)\right],
$$

from which we can read off the dominant effects. At one-loop, the evolution of $\lambda_{t}$ is independent of the $L L \bar{E}$ operators. If we look at equation 2.15, as well as the equations for the two-loop anomalous dimensions $\Gamma_{Q_{3}}^{Q_{3}}, \Gamma_{U_{3}}^{U_{3}}$, and $\Gamma_{\bar{H}}^{\bar{H}}$ we see that for $\lambda_{i j k}^{\prime}=0$, for all $i, j, k$, there is also no dependence on $L L \bar{E}$ at two-loops. Thus $\lambda_{t}\left(M_{U}\right)$ is independent of $\lambda_{323}\left(M_{Z}\right)$ at two-loop, as we see in Fig. 1b.

For $L_{3} Q_{3} \bar{D}_{3}$ we see that $\lambda_{t}\left(M_{U}\right)$ should grow with $\lambda_{333}^{\prime}$. The full two-loop effect is shown in Fig. 2b. At the perturbative limit $\lambda_{t}$ is large but clearly unequal to 1 . The increase is by about $60 \%$. The derivative at $M_{Z}$ does not vanish and we do not observe fix-point structure for $\lambda_{t}$ or $\lambda_{333}^{\prime}$. For lower values of $\tan \beta$ but well above 1.5 new fixed point solutions exist.

For $\bar{U}_{3} \bar{D}_{2} \bar{D}_{3}$ we have a larger positive coefficient than in the previous case and as we see in Fig $3 \mathrm{~b} \lambda_{t}$ rises more quickly as we approach the perturbative limit. For $\lambda_{323}^{\prime \prime}\left(M_{Z}\right) \approx 1, \lambda_{t}$ has the fix-point structure, i.e.

$$
6 \lambda_{t}^{2}\left(M_{Z}\right)+2 \lambda_{323}^{\prime \prime 2}\left(M_{Z}\right) \approx \frac{16}{3} 4 \pi^{2} \alpha_{3}\left(M_{Z}\right) .
$$

This is roughly the same point where $R_{b / \tau} \approx 1$. Thus for $\bar{U}_{3} \bar{D}_{2} \bar{D}_{3}$ bottom-tau unification also corresponds to top IR fix-point structure, as in the MSSM [27, 36, 1]. This is all quite remarkable. The $\mathbb{R}_{p}$-couplings can have significant affects on the entire Yukawa unification picture. From the one-loop RGE for $\lambda_{323}^{\prime \prime}$ we can see that we are well away from its infra-red fixed-point. 


\section{Conclusions}

We have argued that $R_{p}$ is theoretically on equal footing with conserved $R_{p}$. Since it can be realized in grand unified theories it is relevant for unification. We then first determined the complete two-loop renormalization group equations for the dimensionless couplings of the unbroken supersymmetric Standard Model. It is only at two-loop that Yukawa couplings affect the running of the gauge coupling constants. We then considered three models of $R_{p}$. We have added to the MSSM in turn the three Yukawa operators $L_{3} L_{2} \bar{E}_{3}, L_{3} Q_{3} \bar{D}_{3}$, and $\bar{U}_{3} \bar{D}_{2} \bar{D}_{3}$. We considered their effects on various aspects of the perturbative unification scenario. We have focused on qualitative effects. A detailed search for a preferred model is beyond the scope of this paper. We found several important effects. The unification scale is shifted by upto $\pm 20 \%$. This is comprable to some threshold effects but insufficient for string unification. $\alpha_{s}\left(M_{Z}\right)$ can be changed at most by $\pm 5 \%$. The reduction which is favoured by the data is obtained close to the perturbative limit of $\lambda_{333}^{\prime}$. We have obtained the two-loop limit from perturbative unification for all three operators. For $\lambda_{323}$ it is equivalent to the laboratory bound for a slepton mass of $1 \mathrm{TeV}$. For $\lambda_{323}^{\prime \prime}$ the two-loop limit is $15 \%$ weaker than the oneloop limit previously obtained. For bottom-tau unification we have found significant affects. For $L_{3} L_{2} \bar{E}_{3}$ bottom-tau unification can be obtained for values of $\tan \beta<1.5$. For $\bar{U}_{3} \bar{D}_{2} \bar{D}_{3}$ we found a new point of bottom-tau unification at $\tan \beta=5$. It is remarkable that this point also shows top Yukawa infra-red fixed point structure but no $\lambda_{323}^{\prime \prime}$ fixed point structure.

\section{Acknowledgements}

We thank Vernon Barger, Probir Roy and Marc Sher for helpful converstations. H.P. would like to thank J. Gunion for a fruitful collaboration which contibuted to this work. This work was partially supported by the National Science Foundation under grant no. PHY94-07194.

\section{Appendix}

We consider a group $G$ with representation matrices $\mathbf{t}^{A} \equiv(\mathbf{t})_{i}^{A j}$. Then the quadratic Casimir $C(R)$ of a representation $R$ is defined by

$$
\left(\mathbf{t}^{A} \mathbf{t}^{B}\right)_{i}^{j}=C(R) \delta_{i}^{j}
$$

For $S U(3)$ triplets $q$ and for $S U(2)_{L}$ doublets $L$ we have

$$
C_{S U(3)}(q)=\frac{4}{3}, \quad C_{S U(2)}(L)=\frac{3}{4} .
$$

For $U(1)_{Y}$ we have

$$
C(f)=\frac{3}{5} Y^{2}(f)
$$

where $Y(f)$ is the hypercharge of the field $f$. The factor $3 / 5$ is the grand unified normalization.

For the adjoint representation of the group of dimension $d(G)$ we have

$$
C(G) \delta^{A B}=f^{A C D} f^{B C D}
$$


where $f^{A B C}$ are the structure constants. Specifically for the groups we investigate

$$
C\left(S U(3)_{C}\right)=3, \quad C\left(S U(2)_{L}\right)=2, \quad C\left(U(1)_{Y}\right)=0,
$$

and $C(S U(N))=N$. The Dynkin index is defined by

$$
\operatorname{Tr}_{R}\left(\mathbf{t}^{A} \mathbf{t}^{B}\right) \equiv S(R) \delta^{A B} .
$$

For the respective fundamental representations $f$ we obtain

$$
\begin{aligned}
S U(3), S U(2): & S(f) & =\frac{1}{2}, \\
U(1)_{Y}: & S(f) & =\frac{3}{5} Y^{2}(f),
\end{aligned}
$$

where we have inserted the GUT normalization for $U(1)_{Y}$.

The coefficients in the two-loop running of the gauge couplings (2.8) are given by [30]

$$
\begin{aligned}
B_{a}^{(1)} & =\left(\frac{33}{5}, 1,-3\right), \\
B_{a b}^{(2)} & =\left(\begin{array}{ccc}
199 / 25 & 27 / 5 & 88 / 5 \\
9 / 5 & 25 & 24 \\
11 / 5 & 9 & 14
\end{array}\right), \\
C_{a}^{u, d, e} & =\left(\begin{array}{ccc}
26 / 5 & 14 / 5 & 18 / 5 \\
6 & 6 & 2 \\
4 & 4 & 0
\end{array}\right) .
\end{aligned}
$$

\section{References}

[1] V. Barger, M.S. Berger and P. Ohmann, Phys. Rev. D 47 (1993) 1093.

[2] N. Polonsky and P. Langacker, Phys. Rev. D 47 (1993) 4028.

[3] M. Carena, S. Pokorski, and C.E.M. Wagner, Nucl. Phys. B 406 (1993) 59.

[4] J. Ellis, S. Kelley and D.V. Nanopoulos, Phys. Lett. B 249 (1990) 131; P. Langacker, M.X. Luo, Phys. Rev. D 44 (1991) 817; U. Amaldi, W. de Boer and H. Fürstenau, Phys. Lett. B 260 (1991) 447; R. G. Roberts and G. G. Ross, Nucl. Phys. B 377 (1992) 571.

[5] L. Ibanez and G.G. Ross, Nucl. Phys. B 368 (1992) 3.

[6] A. Chamseddine and H. Dreiner, Nucl. Phys. B 447 (1995) 195.

[7] A. Chamseddine and H. Dreiner, ETH-Zürich, ETH-TH-95-04, hep-ph/9504337, to be published in Nucl. Phys. B. 
[8] B. Campbell, S. Davidson, J. Ellis and K. A. Olive, Phys. Lett. B 256 (1991) 457; Astropart. Phys. 1 (1992) 77; W. Fischler, G. Giudice, R. G. Leigh and S. Paban, Phys. Lett. B 258 (1991) 45.

[9] H. Dreiner and G.G. Ross, Nucl. Phys. B 410 (1993) 188.

[10] S. Dimopoulos, R. Esmailzadeh, L. J. Hall, G.D. Starkman, Phys. Rev. D 41 (1990) 2099; H. Dreiner and G.G. Ross, Nucl. Phys. B. 365 (1991) 597; S. Lola, J. McCurry, Nucl. Phys. B 381 (1992) 559; J. Butterworth and H. Dreiner, Nucl. Phys. B 397 (1993) 3; R. M. Godbole, P. Roy, X. Tata, Nucl. Phys. B 401 (1993) 67; H. Dreiner and P. Morawitz, Nucl. Phys. B 428 (1994) 31.

[11] V. Barger, G.F. Giudice, and T. Han. Phys. Rev. D 40 (1989) 2987.

[12] V. Barger, W.-Y. Keung, and R.J.N. Phillips, Phys. Lett. B 356 (1995) 546.

[13] B. Brahmachari, P. Roy, Phys. Rev. D 50 (1994) 39, ERRATUM ibid D (1995) 51.

[14] J.L. Goity and M. Sher, Phys. Lett. B 346 (1995) 69.

[15] C. E. Carlson, P. Roy, M. Sher, Phys. Lett. B 357 (1995) 99.

[16] A. Yu. Smirnov and F. Vissani, ICTP, Trieste IC-95-122, hep-ph/9506416.

[17] R. Hempfling, Max Planck Inst. MPI-PHT-95-59, hep-ph/9511288.

[18] G. Bhattacharyya, J. Ellis and K. Sridhar, Mod. Phys. Lett. A 10 (1995) 1583.

[19] L. J. Hall and M. Suzuki, Nucl. Phys. B 231 (1984) 419.

[20] D. E. Brahm and L. J. Hall, Phys. Rev. D 40 (1989) 2449.

[21] M.C. Bento, L. J. Hall, and G.G. Ross (Oxford U.). Nucl. Phys. B 292 (1987) 400.

[22] M. Chanowitz, J. Ellis and M. Gaillard, Nucl. Phys. B 128 (1977) 506; A. Buras, J. Ellis, M. Gaillard and D.V. Nanopoulos, ibid. B 293 (1978) 66; M.B. Einhorn and D.R.T. Jones, ibid. B 196 (1982) 475; J. Ellis, D.V. nanopoulos and S. Rudaz, ibid. B 202 (1982) 43; J. Ellis, S. Kelley, and D.V. Nanopoulos, Nucl. Phys. B 373 (1992) 55.

[23] H. Arason, D.J. Castano, B. Keszthelyi, S. Mikaelian, E.J. Piard, P. Ramond and B. D. Wright, Phys. Rev. Lett. 67 (1991) 2933.

[24] CDF Collaboration, F. Abe et al., Phys. Rev. Lett. 74 (1995) 2626; D0 Collaboration, S. Abachi et al., Phys. Rev. Lett. 74 (1995) 2632. 
[25] Savas Dimopoulos, Lawrence J. Hall, Stuart Raby Phys. Rev. Lett. 68 (1992) 1984; Phys. Rev. D 45 (1992) 4192; G. Giudice, Mod. Phys. Lett. A 7 (1992) 2429; J. Harvey, P. Ramond and D. Reiss, Phys. Lett. B 92 (1980) 309; Nucl. Phys. B 199 (1982) 223; H. Dreiner, G.K. Leontaris, N.D. Tracas, Mod. Phys. Lett. A8 (1993) 2099; P. Ramond, R.G. Roberts, G.G. Ross, Nucl. Phys. B 406 (1993) 19.

[26] L. Ibanez and G.G. Ross, Phys. Lett. B 332 (1994) 100, and references therein.

[27] B. Pendleton and G.G. Ross, Phys. Lett. B 98 (1981) 291; C.T. Hill, Phys. Rev. D 24 (1981) 691.

[28] W.A. Bardeen, M. Carena, T.E. Clark, C.E.M. Wagner, and K. Sasaki, Nucl. Phys. B369 (1992) 33; M. Carena, M. Olechowski, S. Pokorski, and C.E.M. Wagner, Nucl. Phys. B 419 (1994) 213.

[29] S.P. Martin and M.T. Vaughn, Phys. Rev. D 50 (1994) 2282.

[30] J.E. Björkman and D.R.T. Jones, Nucl. Phys. B 259 (1985) 533.

[31] J. Gunion, D. McKay and H. Pois, UC, Davis. UCD-95-18, hep-ph/9507323.

[32] J. Gasser and H. Leutwyler, Phys. Rep. 87 (1982) 77 .

[33] N. Polonsky and P. Langacker, Phys. Rev. D 50 (1994) 2199.

[34] Fig 5 of Ref. [1]; Figs 2 and 3 of Ref. [3].

[35] Particle Data Group, Phys. Rev. D 50 (1994) 1173.

[36] W. Bardeen, M. Carena, S. Pokorski, and C. Wagner, Phys. Lett. B 320 (1994) 110; J. Bagger, S. Dimopoulos and E. masso, Phys. rev. Lett. 55 (1985) 920.

Figure 1: We have fixed the values $\tan \beta=5, m_{b}\left(m_{b}\right)=4.25 \mathrm{GeV}$ and $m_{t}\left(m_{t}\right)=165 \mathrm{GeV}$. For a vanishing top-quark Yukawa coupling and a vanishing $\mathbb{R}_{p}$-coupling we obtain the unification values shown in Fig (1a). In (1a) we then show the ratios $R_{M_{U}}, R_{\alpha_{U}}$ and $R_{\alpha_{3}}$ at the unification scale as a function of $\left(\Lambda_{E^{3}}\right)_{23}\left(M_{Z}\right)$. In Fig. (1b) we show the values of $R_{b / \tau}$, $\lambda_{t}$ and $\left(\Lambda_{E^{3}}\right)_{23}$ at the unification scale as a function of $\left(\Lambda_{E^{3}}\right)_{23}\left(M_{Z}\right) .\left(\Lambda_{E^{3}}\right)_{23}\left(M_{U}\right)$ reaches its perturbative limit for $\left(\Lambda_{E^{3}}\right)_{23}\left(M_{Z}\right)=0.9$. At this point it is outside the plotted region. The plotted region in Figs (a) and (b) is chosen so as to highlight the evolution of the other quantities.

Figure 2: The same as Figure 1 just for $\left(\Lambda_{D^{3}}\right)_{33}$ except the perturbative limit is reached for $\left(\Lambda_{D^{3}}\right)_{33}\left(M_{Z}\right)=1.08$.

Figure 3: The same as Figure 1 just for $\left(\Lambda_{U^{3}}\right)_{23}$ except the perturbative limit is reached for $\left(\Lambda_{U^{3}}\right)_{23}\left(M_{Z}\right)=1.14$. 
This figure "fig1-1.png" is available in "png" format from: http://arxiv.org/ps/hep-ph/9511444v1 
This figure "fig1-2.png" is available in "png" format from: http://arxiv.org/ps/hep-ph/9511444v1 
This figure "fig1-3.png" is available in "png" format from: http://arxiv.org/ps/hep-ph/9511444v1 